\title{
UV Fluorescence Imagery of the Turin Shroud - Digitally Revisited
}

\author{
Samuel Pellicori \\ Pellicori Optical Consulting, Santa Barbara, USA
}

Email address:

pellopt@cox.net

\section{To cite this article:}

Samuel Pellicori. UV Fluorescence Imagery of the Turin Shroud - Digitally Revisited. International Journal of Archaeology. Vol. 8, No. 2, 2020, pp. 32-36. doi: 10.11648/j.ija.20200802.13

Received: December 2, 2020; Accepted: December 14, 2020; Published: December 22, 2020

\begin{abstract}
UV fluorescence imagery of faint or fragile images and markings (writing) on relics and artwork is an affordable non-destructive tool useful in revealing often invisible details and in monitoring temporal stability. UV fluorescent images of Turin were recorded for the first time during the 1978 scientific investigation. The original images were recorded on color film and now have been digitally scanned and enhanced using image processing software. The processed UV images contain spectral discriminatory information and high spatial detail resolution with high contrast that is not discernable in white light images. Differing fluorescent emission colors were found to be associated with image features such as body image, burns, blood flows, skin wounds and water flows on the Turin Shroud. Spectral signature information has the potential for assisting the determination or elimination of the causes responsible for the appearance of the various features. Inconsistent and nonuniform exposure problems in the original photography were revealed, and their impact the subsequent digitization of the images is discussed. Suggestions for future fluorescence image collection include the design of equipment that will eliminate the problems associated illumination and film exposure. The extraction of spectral and spatial detail through UV fluorescence imagery is relevant to tracking temporal and climatic changes to assist conservation efforts.
\end{abstract}

Keywords: UV Fluorescence Studies, Turing Shroud, Conservation Efforts.

\section{Introduction}

Fluorescent emission stimulated by UV energy is a nondestructive diagnostic tool that has application in art forgery detection and conservation, chemical reaction product identification, mineralogical composition, bio-medical diagnostics, crime forensics, surface contamination and chemical dye and paint detection. Emission occurs at visible wavelengths and requires high levels of isolation and separation of the stimulating and emitting wavelength energies.

Many publications have presented the known historicity of the Turin shroud [1-4]. In 1978, multidisciplinary scientific studies were performed on the Turin Shroud by a team of specialists employing optical physics, biochemical and multispectral spectroscopic techniques [1-3]. The objective of the scientific study performed by Shroud of Turin Research Project (STRUP) was to attempt to determine and/or eliminate the possible causes for the appearance of the image of the body of a human male on the $4.3 \mathrm{~m} \mathrm{x} 1.1 \mathrm{~m}$ linen cloth. The dorsal and frontal images have the unique quality of not being the product of applied paints, powders, dyes, or other pigments. The mechanism of the image generation remains a subject of research [1-3].

A second objective of the 1978 investigation was to suggest conservation procedures to prevent progressive contrast dilution of the image. The concern is that through natural aging, the linen's yellow color density is intensifying to the degree that the visible contrast between image and cloth background is being lost. Images recorded in blue (or short wavelength) light show higher contrast than images collected at longer wavelengths because material (specifically cellulose) absorption coefficients increase toward shorter wavelengths.

UV fluorescence imaging reveals features that have even higher absorption, and therefore higher contrast, as well as individual spectral fluorescent signatures. UV fluorescence (UVF) photography was performed for the first time during the 1978 scientific investigation by the late Vernon Miller and was film-based at the time of the investigation [5]. The objective of this report is to make digitized versions of that 
historic photography available.

Visual reflected light photography of the Turin Shroud reveals nearly monochromatic images with low color hue and density contrasts against the clear cloth background. The faint sepia color of the cloth and the image suggested that imagery at short wavelengths, specifically deep blue and UV wavelengths shorter than $\sim 420 \mathrm{~nm}$, would enhance the contrast of features and markings because they would selectively absorb short wavelengths more strongly than the cloth background. When the visible wavelengths are filtered out to record imagery in the short wavelength (blue) region, the contrast between the body image and the background cloth was found to be higher.

Fluorescence stimulation by UV was introduced as a method to enhance the spectral emission and absorption differences of the various marks and features, and to produce information that might be useful in suggesting or eliminating possible causative mechanisms and further be useful for future conservation procedures. There is a suspicion that overtime, the yellow color of the background cloth is gradually darkening with respect to the body image thus further reducing the contrast. Until the 1978 investigation, little attention was paid to proper conservation efforts during storage, handling, and public exhibition.

\section{UV / Visible Image Isolation}

Original UV fluorescence exposures were made on Kodacolor 400 color negative film with a Hassleblad EL camera in a 2-1/4-inch format size [5]. The color negative film recorded fluorescence emissions at wavelengths between $\sim 350 \mathrm{~nm}$ and $\sim 700 \mathrm{~nm}$ that were excited by a $40 \mathrm{~nm}$ wide UV passband centered at $355 \mathrm{~nm}$. Limiting excitation to UV and recording only visible emitted light required the design and construction of a custom UV excitation filter with high rejection of longer wavelengths. The UV filter technology of the day was based on absorption-type filters to reject long wavelengths and were constructed of Corning 7-54 UVtransmitting filter glass and a $1 \mathrm{~cm}$ path of an aqueous solution of cobalt-sulfate + copper-sulfate + nickel-sulfate to remove the leak above $\sim 600 \mathrm{~nm}$ that the filter glass transmits. Figure 1 shows the transmittance of the custom designed excitation filter.

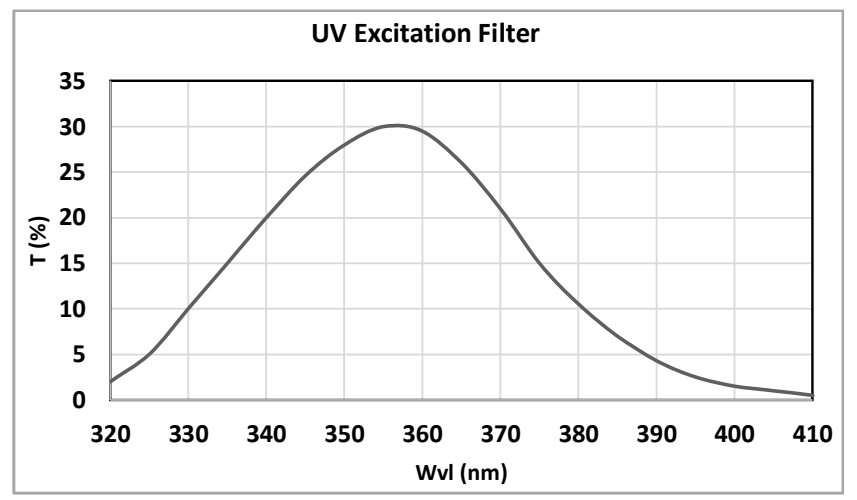

Figure 1. Transmittance passband of the liquid UV-transmitting filter:
Identical $15-\mathrm{cm}$ diameter excitation filters were mounted to the reflectors of two 200 watt-second xenon strobe lamps mounted at opposite $45^{\circ}$ incidence directions and illuminated the shroud in a plane oriented in the long $(4 \mathrm{~m})$ dimension. The camera and UV source assembly were moved on a rail to image different sections of the shroud [5]. A mosaic of eight $53.3 \mathrm{~cm}$ square camera fields were photographed perpendicular to the cloth over the $4.3 \times 1.1 \mathrm{~m}$ shroud area. The UV passband exhibited no angle-dependence. At longer wavelengths outside the passband, transmission dropped to $<0.1 \%$ at $435 \mathrm{~nm}$ and averaged $<0.01 \%$ to $\sim 700 \mathrm{~nm}$. This liquid filter technology was adapted from astronomical photometry of stars and planets that used the NUBV photometric system, where the $\mathrm{N}$ filter had a $360 \mathrm{~nm}$ CWL UV passband [6]. The camera was filtered with a Hoya L-42 UV-absorbing glass filter that transmitted visible light and removed all energy at wavelengths shorter than 400 $\mathrm{nm}$ [5]. It also prevented potential background contaminating fluorescence from the camera lens. Test exposures using a simulated shroud were made by at the former Brooks Institute of Photography in Santa Barbara before the equipment was shipped to Turin, Italy.

\section{Digital Retrieval of the Fluorescence Images}

Miller provided the author with a set of $35 \mathrm{~mm}$ transparencies made from the original $4 \times 5$ inch color negatives. The individual images were processed using the image analysis and processing software in Adobe Photoshop 2021.

Unlike luminous photography, there are no color references for fluorescence imagery, so the reproduction of the true fluorescing hues is subject to error. We circumvented that limitation by using the photoelectric spectrophotometry measurements made during the 1978 examination as a reference [7]. A dual grating spectrometer recorded the emission spectral of various features and of the cloth background in several areas. UVF colors that were stimulated by the $365 \mathrm{~nm}$ emission line of a filtered mercury lamp ("black light") were observed and provided color reference points. It was visually noted that the background cloth fluoresced with a yellow-green hue, faint scorches emitted a reddish-brown hue while the body and blood features emitted no fluorescence.

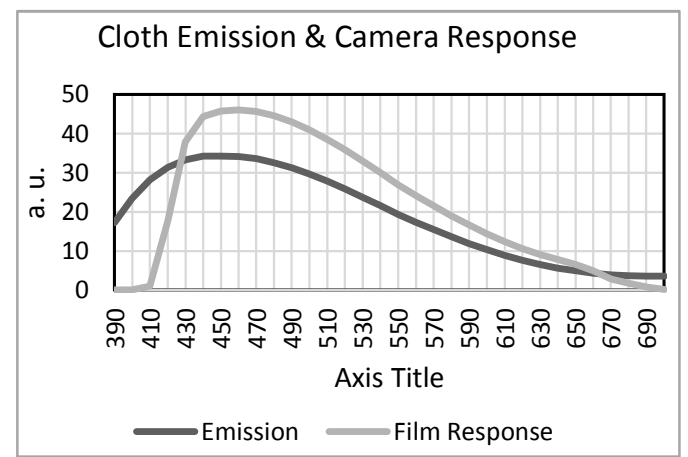

Figure 2. Photoelectric measurement of the fluorescence emission from the average cloth background [7] and the spectral response of the camera with the UV blocking filter. 
The photoelectric data was used in the simulation of the mean background emission curve as illuminated with an equal-energy distribution and the resulting visual color sensation was generated using the thin-film design and analysis program, Essential Macleod [8]. The curves in Figure 2 are the background emission of clear cloth areas and the response of the camera. The simulated visual hue of the clear cloth background spectral emission is greenish blue.

Reference background fluorescent hue appears in the original published photos [5]. In the photographic reproduction, the background fluorescent hue was used together with black burned areas in the frames to bound the hue spectrum in each image. Black was present either in the border areas outside the image or in charred areas in the image.

It is a challenge to uniformly illuminate an area as large as $53 \mathrm{~cm}$ on a side. The problem is more severe when working with UV wavelengths. Complicating the reproduction of the 1978 UVF images was illumination non-uniformity that varied over a range as high as 3 to 1 between center and edges (burned areas), Figure 3. The two strobed Xenon flash lamps were aimed at $45^{\circ}$ to the photographed area in the plane of the long dimension of the TS. The Norman 202 strobe units were slaved to the camera shutter. The Shroud was mounted with its $4.3 \mathrm{~m}$ dimension in the horizontal axis. Figure 3 has a typical horizontal distribution profile measured just above the patches of Figure 4 . The peak-tovalley non-uniformity in the vertical $(1.1 \mathrm{~m})$ direction was $<5 \%$. Another complication that affected the color reproduction was overexposure of some images that was attributed to the unregulated and therefore inconsistent charging and discharging of the strobe's capacitors. Attempts were made using software to reduce the non-uniformity and overexposure effects without significantly affecting the relative distinctive hues. This task proved to be challenging. In some cases where the image was overexposed or severely affected by the illumination fall off at the edges, applied brightness correction modified the hues. In the interest of emphasizing the emission differences, the approach taken was to optimize feature visibility differences in deference to the accurate reproduction of the background greenish-blue fluorescence hue.

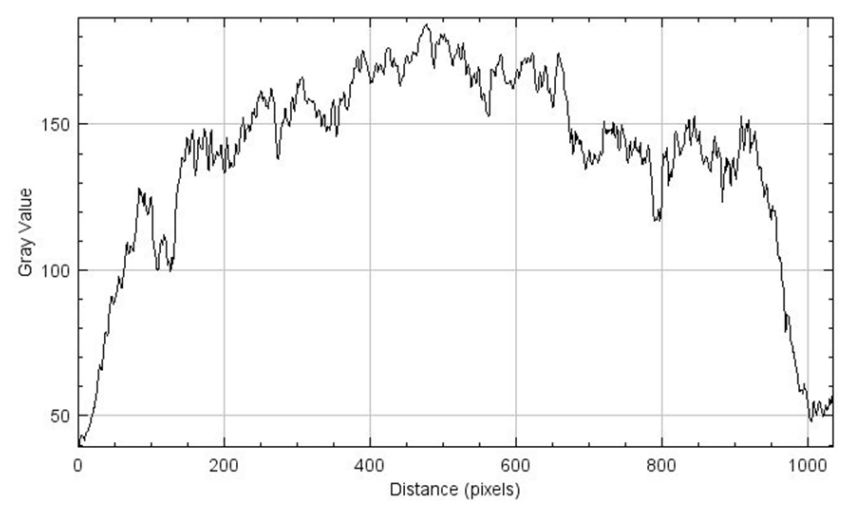

Figure 3. Fluorescing intensity profile along the $4.3 \mathrm{~m}$ (long) axis that contained the two strobe lights at 45 . Traced just above the patches in Figure 4.
Examples of fluorescing signatures of materials and markings recorded on the visible-responding color film are shown in Figures 4-6. Figures 4-6 show white-light and corresponding fluorescent images of selected areas of the TS that were digitally processed using Adobe Photoshop 2021. Processing consisted of blanketing brightness and contrast enhancement of the whitelight and UVF images including minor color adjustments to produce black in burned areas. The higher contrast of the marks and features in the UVF images indicate larger optical absorption in body markings, blood, and water stains relative to the background cloth. The cloth background emits a faint greenish hue in approximate agreement with the spectral distribution measured photoelectrically. Different fluorescing colors can be seen for the various markings. Features such as the faint scorches and body image areas appear in visible images to have equal optical density and color. When stimulated by UV of wavelengths near $360 \mathrm{~nm}$ the faint scorches fluoresce a faint reddish hue. The body image emits no color; in fact, it absorbs the fluorescence of the cloth background rendering it neutral in color. The differential emitted fluorescent colors and contrasts of the various markings of were hypothesized to relate to the mechanisms responsible for the origins of the various emissions [1-3]. The inhomogeneous weave structure of the cloth is resolved, apparently indicative of different thread composition or chemical processing. Patches sewn on in A. D. 1534 to cover the burned areas caused by the fire of 1532 fluoresce brighter and with different colors, indicating that they are of different compositions and ages than the background cloth.

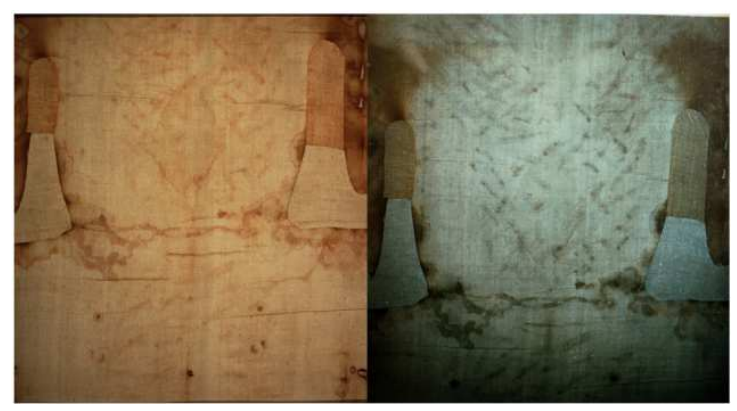

Figure 4. Dorsal image area. Left: white light, right: UVF. Background cloth fluoresces a greenish tint. The skin wounds on the back are more visible in the UVF photo and the blood flow is differentiated between solids and serum and has a different tint than the body image. Faint scorches at the patches emit a reddish hue.
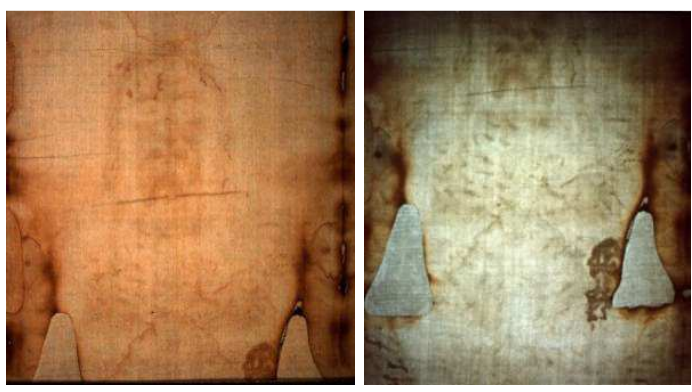

Figure 5. Face and chest area. Left: white light, right: UVF. Background cloth fluoresces a greenish tint. Higher spatial detail is revealed in the UVF photo. Image features are dark, indicating absorption while faint scorches emit reddish hue. The water stain between the patches is outlined by a waterfront (border) where transported solids were deposited. 


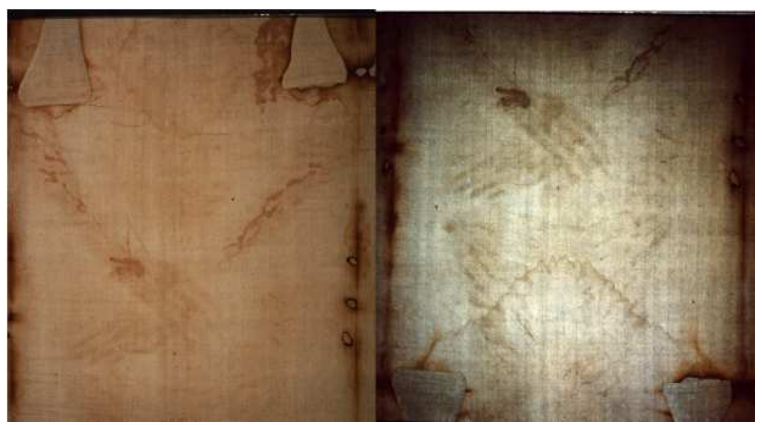

Figure 6. Hands and upper legs area. Left: white light, right: UVF. Hands and body areas are absorbing (rather than fluorescing) as is the triangular waterfront. Faint scorches covered by the patches fluoresce reddish.

Color discrimination and spatial resolution are higher in the UVF images. Adventitious stains are evident over the entire cloth. The fluorescing images exhibit weave and density irregularities in the cloth. Further images are available on www.shroudphotos.com.

\section{Future UVF and Conservation Efforts}

The unique image and associated markings on the Turin Shroud and any similar antiquities are in danger of being lost unless conservation efforts are considered.

There are many ways to generate chromophores in linen; that are the reaction products of dehydration, oxidization and conjugation of the cellulose molecule [1-3]. The rate of the natural degradation process is dependent on exposure parameters such as heat, humidity and solar UV, and can be accelerated by the presence of foreign surface materials [9]. Cellulostic materials such as wood and paper and potentially volatile emitting polymer materials should not be used to contain or store the TS.

Changes in the fluorescent properties of organic materials such as blood components can be induced by UV, humidity and temperature changes. Cellulose can combine with water in two forms, volatile and bound. The volatile component can change with changing climate environment. Both the mechanical and optical properties are therefore dependent on the external humidity [10]. Combined with UV exposure and temperature changes, cellulose can degrade at accelerated rates. UV exposure of humid cellulose can activate chemical reactions that produce absorption of blue light leaving a yellow tint. Therefore, it is important to maintain a constant, non-zero humidity and its associated temperature. The optimum climatic conditions for conserving the TS image have not been decided, however parameters for comparable antiquities suggest that the relative humidity should be $\sim 40 \%$ at $20 \mathrm{C}$ at ambient pressure. Vacuum or a pure nitrogen atmosphere should be avoided. Dry nitrogen gas is an efficient dehydrating agent. $\mathrm{RH}>70 \%$ encourages the growth of molds and fungus. [11. 12]

Typical glass transmits to $\sim 320 \mathrm{~nm}$ therefore, additional protective filtering is required to eliminate wavelengths shorter than $\sim 420 \mathrm{~nm}$. Exposure to high-energy light such as UV at wavelengths $<\sim 420 \mathrm{~nm}$ should be avoided. In the 1978 investigation, UV irradiation was provided by two $200 \mathrm{~W}$-sec Xenon strobe sources. Irradiation intensity at the Shroud surface from the strobes was not measured, however, the flash times were $<50 \mathrm{~ms}$ in duration. The irradiance in the passband in a 50 $\mathrm{ms}$ flash was estimated to be $<20 \mathrm{~mW} / \mathrm{m}^{2}$ at $1 \mathrm{~m}$ distance. This compatible with the conservator's guideline of $<1.5 \mathrm{~mW} / \mathrm{m}^{2}$ for continuous exposure $[13,14]$.

Future UVF would use a mid-format DSLR camera and uniform stimulating irradiation produced by arrays of pulsed $365 \mathrm{~nm}$ LEDs. A LWP filter on the camera would define the UV cut-off edge and eliminate possible fluorescence in the lens while transmitting the visible emissions. Camera exposures can be adjusted in real time. White light photography of the same areas would be registered to the UVF images. The collected digital data files permit accurate color reproduction and discrimination and be used to monitor and quantify changes, thus providing historically relevant feedback for conservation efforts.

\section{Funding}

Pellicori Optical Consulting

\section{Acknowledgements}

The assistance of Mike Verbois and Barrie Schwortz, both of whom are former students of Vernon Miller and assisted in the 1978 investigation, is appreciated.

\section{Disclosures}

The author declares no conflicts of interest.

\section{References}

[1] Jumper, Eric J., Alan D. Adler, John P. Jackson, Samuel F. Pellicori, John H. Heller, James R. Druzik, "A Comprehensive Examination of the Various Stains and Images on the Shroud of Turin", ACS Advances in Chemistry No. 205. Archaeological Chem. III, American Chemical Society 22, 447-476 (1984).

[2] L. A. Schwalbe and R. N. Rogers Analytica Chimica acta, 135, 3-49 (1982) printed in the Netherlands Special Report Physics and Chemistry of the Shroud of Turin a Summary of the 1978 Investigation.

[3] Eric J. Jumper and Robert W. Mottern, Applied Optics, Vol. 19, 1909-1912 (1980).

[4] Ian Wilson, The Mysterious Shroud, Doubleday \& Company, Garden City New York, 1986, ISBN 0-385-19074-3.

[5] Miller, V. D. and Pellicori, S. F.: "Ultraviolet Fluorescence Photography of the Shroud of Turin", J. Biological Photographers Assoc. 49, No. 3, 71-85 (1981). available from www.shroud.com.

[6] S. F. Pellicori, "Transmittances of Some Optical Materials for use Between 1900 and 3400 A", Appl. Opt, 3 no. 5, 361-36 (1964). 
[7] Roger Gilbert, Jr., and Marion M. Gilbert, "Ultraviolet-visible reflectance and fluorescence spectra of the Shroud of Turin", Applied Optics, Vol. 19, page 1930-1936 (1980).

[8] Essential Macleod Thin Film Software, www.thinfilmcenter.com.

[9] Pellicori, S. F.: "Spectral Properties of the Shroud of Turin", Appl Opt. 19, 1913-1920 (1980).

[10] Alan D. Adler and Larry A. Schwalbe, "Conservation of The Shroud of Turin", Shroud Spectrum International, No. 42, December (1993).

[11] Stephen Brown, "Guidelines for Environmental Control in Cultural Institutions", Consortium for Heritage Collections and their Environment, (2002). www.researchgate.net/publication/341026505
[12] Jo Kirby Atkinson, "Environmental conditions for the safeguarding of collections: A background to the current debate on the control of relative humidity and temperature", Studies in Conservation, (2014) 59: 4, 205-212, DOI: $10.1179 / 2047058414$ Y.0000000141.

[13] Stefan Michalski, "Measurement of Ultraviolet Radiation Canadian Conservation Institute (CCI) Notes 2/2. ISBN ISSN 1928-1455.

http://opensi.si.edu/index.php/smithsonian/catalog/book/111

[14] Michalski, S. "Damage to Museum Objects by Visible Radiation (Light) and Ultraviolet Radiation (UV)." In, Lighting in Museums, Galleries and Historic Houses. London: Museums Association, UKIC, and Group of Designers and Interpreters for Museums, 1987, pp. 3-16. 